\title{
Ocular Rotation Axes during Dynamic Bielschowsky Head-Tilt Testing in Unilateral Trochlear Nerve Palsy
}

\author{
Konrad P. Weber, ${ }^{1}$ Klara Landau, ${ }^{2}$ Antonella Palla,${ }^{1}$ Thomas Haslwanter, ${ }^{1}$ and \\ Dominik Straumann ${ }^{1}$
}

Purpose. To explain the positive Bielschowsky head-tilt (BHT) sign in unilateral trochlear nerve palsy (UTNP) by the kinematics of three-dimensional eye rotations.

Methods. Twelve patients with uTNP monocularly fixed on targets on a Hess screen were oscillated $\left( \pm 35^{\circ}, 0.3 \mathrm{~Hz}\right)$ about the roll axis on a motorized turntable (dynamic BHT). Threedimensional eye movements were recorded with dual search coils. Normal data were collected from 11 healthy subjects.

RESULTS. The rotation axis of the viewing paretic or unaffected eye was nearly parallel to the line of sight. The rotation axis of the covered fellow eye, however, was tilted inward relative to the other axis. This convergence of axes increased with gaze toward the unaffected side. Over entire cycles of head roll, the rotation axis of either eye remained relatively stable in both the viewing and covered conditions.

Conclusions. In patients with uTNP, circular gaze trajectories of the covered paretic or unaffected eye during dynamic BHT are a direct consequence of the nasal deviation of the rotation axis from the line of sight. This, in turn, is a geometrical result of decreased force by the superior oblique muscle (SO) of the covered paretic eye or, according to Hering's law, increased force parallel to the paretic SO in the covered unaffected eye. The horizontal incomitance of rotation axes along horizontal eye positions can be explained by the same mechanism. (Invest Ophthalmol Vis Sci. 2004;45:455-465) DOI:10.1167/ iovs.02-1223

$\mathrm{O}$ scillation of the head in the roll plane leads to conjugate ocular counterroll through the vestibulo-ocular reflex. ${ }^{1,2}$ If a subject binocularly fixes on a visual target during this torsional vestibular stimulation, the ocular rotation axis of either eye aligns with the line of sight to maintain fixation, ${ }^{3}$ whereas in the dark, the ocular rotation axis tilts into the opposite direction by half the angle whenever the line of sight is directed eccentrically. ${ }^{4}$ During monocular fixation, with the

From the ${ }^{1}$ Department of Neurology, Zurich University Hospital, Zurich, Switzerland; ${ }^{2}$ Department of Ophthalmology, Zurich University Hospital, Zurich, Switzerland.

Supported by Swiss National Science Foundation Grant 32-51938.97 SCORE A (DS), 31-63465.00 (DS), and 3100-063669 (TH); and the Betty and David Koetser Foundation for Brain Research, Zurich, Switzerland.

Presented in part at the meeting of Physiology and Disorders of Oculomotor and Vestibular Control, Wildbad Kreuth, Germany, April 2003.

Submitted for publication November 28, 2002; revised June 7 and October 6, 2003; accepted October 26, 2003.

Disclosure: K.P. Weber, None; K. Landau, None; A. Palla, None; T. Haslwanter, None; D. Straumann, None

The publication costs of this article were defrayed in part by page charge payment. This article must therefore be marked "advertisement" in accordance with 18 U.S.C. $\$ 1734$ solely to indicate this fact.

Corresponding author: Dominik Straumann, Department of Neurology, Zurich University Hospital, Frauenklinikstrasse 26, CH-8091, Zurich, Switzerland; dominik@neurol.unizh.ch. head not moving, the line of sight of the covered eye points toward the same target as the viewing eye, in accordance with Hering's law of equal innervation. ${ }^{5}$ This is also observed during concurrent torsional vestibular stimulation, as the ocular rotation axis aligns with the line of sight in both eyes. ${ }^{6}$

In trochlear nerve palsy, the line of sight of the covered eye no longer points toward the target, but deviates up- or downward when the paretic or healthy eye is covered, respectively. As a consequence of Hering's law, this torsional-vertical deviation is incomitant-that is, it increases when gaze is moved in the pulling direction of the paretic superior oblique muscle by vertical (evoked by visual targets) or torsional (evoked by ocular counterroll) eye displacements. Based on this observation, Bielschowsky and Hofmann ${ }^{7}$ described the head-tilt test for diagnosing trochlear nerve palsies. Rolling the head toward the side of the paretic eye leads to an increase of vertical deviation between the two eyes. In clinical use, this test is performed in a static fashion, comparing the vertical deviation on head roll to both sides.

The change in torsional-vertical deviation between the covered and the viewing eye (torsional-vertical incomitance) during Bielschowsky head-tilt testing requires that the ocular rotation axis of the covered eye is no longer aligned with the line of sight. Otherwise, no vertical incomitance could be observed as a function of ocular counterroll.

We asked whether the orientation of ocular rotation axes that leads to the pattern of binocular deviations observed during Bielschowsky head-tilt testing could be predicted by the anatomy of the paretic superior oblique muscle. Three-dimensional eye positions evoked by static head roll, however, are not sufficient to trigonometrically reconstruct the exact orientation of ocular rotation axes, because the amount of ocular torsion is relatively small and fluctuates considerably. ${ }^{8}$ We therefore used continuous sinusoidal vestibular stimulation about the naso-occipital axis and analyzed whether the orientation of ocular rotation axes would change as a function of ocular counterroll. By presenting targets at different locations on a head-fixed Hess screen, ${ }^{9}$ we also tested whether ocular rotation axes would change with eye position and whether there was incomitance between the rotation axes of both eyes, which would extend Hering's law for binocular eye positions to binocular rotation axes.

\section{Methods}

\section{Subjects}

We tested 12 patients (age 15-57 years, two female) with untreated unilateral trochlear nerve palsy. The clinical diagnosis of trochlear nerve palsy was based on the three-step procedure described by Parks, ${ }^{10}$ including Bielschowsky head-tilt testing. ${ }^{7}$ In all patients, intermittent or constant vertical double vision was present for more than 6 months. The comparison group consisted of 11 healthy subjects (age 21- 40 years, 6 women). Informed consent was obtained from patients and healthy subjects after the experimental procedure was explained. The protocol was approved by a local ethics committee and was in 
accordance with the ethical standards laid down in the Declaration of Helsinki for research involving human subjects.

\section{Setup}

Subjects were seated upright on a turntable with three servocontrolled motor driven axes (prototype built by Acutronic, Jona, Switzerland). The head was restrained with an individually molded thermoplastic mask (Sinmed BV, Reeuwijk, The Netherlands) and was positioned such that the center of the interaural line was at the intersection of the three axes of the turntable. Pillows and safety belts minimized movements of the body. Movements of both eyes were recorded in three dimensions ${ }^{11}$ (horizontal, vertical, torsional) on the turntable. Subjects monocularly fixed on nine laser dots projected on a spherical screen at a distance of $1.4 \mathrm{~m}$, whereas the other eye was covered. Healthy and paretic eye viewing conditions were intermingled.

The dots were located straight ahead and at eight eccentric headfixed positions-that is, on a $0^{\circ} \pm 20^{\circ}$ horizontal and vertical square grid in stereographic coordinates. Dot positions were calibrated by rotations of a laser placed in the center of the turntable. For secondary dot positions, the laser was rotated about the horizontal or vertical axis by $20^{\circ}$ from the straight-ahead position. For tertiary dot positions, the laser was rotated about oblique axes $\left(45^{\circ}\right.$ between horizontal and vertical) by $28.3^{\circ}$ from the straight-ahead position.

Three-dimensional eye movements were binocularly recorded with dual search coils manufactured by Skalar (Delft, The Netherlands). The coils were mounted on both eyes after anesthetizing the conjunctiva and cornea with oxybuprocaine hydrochloride $0.4 \%$ (Novartis Ophthalmics, Hettlingen, Switzerland). A chair-fixed coil frame (side length, $0.5 \mathrm{~m}$ ) that produced three orthogonal magnetic fields with frequencies of 80,96 , and $120 \mathrm{kHz}$ surrounded the subject's head. Subjects were seated inside the frame so that the center of the interpupillary line coincided with the center of the frame. The signals were amplified and multiplexed before passing through the turntable slip rings. A high performance 12-bit digital signal processor computed a Fast Fourier transform in real time on the digitized search coil signal to determine the voltage induced in the coil by each magnetic field (system manufactured by Primelec, Regensdorf, Switzerland). Dual search coils were calibrated in vitro on a gimbal system before each experiment. Details of the procedure are given elsewhere. ${ }^{12}$ The orientation of the coil could be determined with an error of less than $7 \%$ over a range of $\pm 30^{\circ}$ and with a noise level of less than $0.05^{\circ}$ (root mean square deviation). Eye- and chair-position signals were digitized with 16-bit accuracy. All data were sampled at $1 \mathrm{kHz}$ and analyzed offline with statistical software (MatLab; The MathWorks, Inc., Natick, MA).

\section{Experimental Procedure}

One eye was covered at least 5 minutes before measurements to break the fusional reflex between the two eyes. First, binocular eye positions during the static Bielschowsky head-tilt test ${ }^{7}$ were recorded with either eye covered in upright, $35^{\circ}$ left ear down, and $35^{\circ}$ right ear down whole-body roll position. In each static turntable position, the subjects sequentially fixed on the nine head-fixed Hess screen targets during 3 seconds each. This was repeated with the other eye viewing. Then, patients were oscillated about the naso-occipital (roll) axis $\left( \pm 35^{\circ}, 0.3\right.$ $\mathrm{Hz}$ ). During this dynamic paradigm, subjects had to fix on the nine head-fixed Hess screen targets during 18 seconds each. Thus, eye position data of five oscillation cycles could be obtained for every gaze direction with either eye covered (see video, Bielschowsky.mov, Appendix B).

\section{Data Analysis}

Detailed explanations and corresponding equations are given in Appendix A. Three-dimensional eye positions in the magnetic coil frame were expressed as rotation vectors. ${ }^{13}$ A rotation vector $\mathbf{r}^{\mathrm{e}}$ describes the instantaneous orientation of the eye as a single rotation from the reference position looking straight ahead. The rotation vector $\mathbf{r}^{\mathrm{e}}$ is oriented parallel to the axis of this rotation, and its length is defined by $\tan (\rho / 2)$, where $\rho$ is the angle of rotation. The signs of rotation vectors are determined by the right-hand rule-that is, clockwise, downward, and leftward rotations, as seen from the subject, are positive. From rotation vectors, angular velocity vectors $\omega$ were derived to determine the rotation axes. ${ }^{14}$ Angular velocity vectors point along the instantaneous rotation axis. Their length is proportional to the rotational speed.

Gaze vectors were projected stereographically ${ }^{13}$ on a Hess screen chart. This chart represents gaze direction from the patient's point of view. Because the stereographic projection is conformal, local angles are preserved and circular gaze movements about a fixed axis appear equally circular. (Note that this is different from rotation vector space, in which a rotation about a single axis is represented by a straight line.)

To relate ocular rotation axes with the corresponding gaze trajectories on the same Hess screen chart, the orientations of the angular velocity vectors (representing ocular rotation axes) were also projected stereographically.

The nine data clouds of gaze directions associated with fixations of the nine Hess screen targets by the viewing eye were selected with an interactive computer program. In patients with left-sided trochlear nerve palsy, the directions of three-dimensional eye position were horizontally mirrored, as if the right eye had been affected by the palsy. In the static paradigm, the median three-dimensional rotation vectors of both eyes were computed for each gaze direction. In the dynamic paradigm, torsional saccades were eliminated from the angular velocity data by iterative sinusoidal fitting.

\section{Computer Simulations}

Simulations to predict eye positions based on geometric considerations were written in commercial software (MatLab; The MathWorks). We also checked whether the recorded three-dimensional eye position data in patients could be predicted by a current biomechanical software model, EyeLab 2000, ${ }^{15}$ based on Orbit version $1.5^{16}$ and written in MatLab.

\section{Results}

\section{Experimental Findings}

Figure 1 depicts a typical example of static Bielschowsky headtilt testing in a patient with congenital trochlear nerve palsy. In the Hess screen projection, the right covered paretic eye (circles connected with solid lines) showed the characteristic pattern of vertical-horizontal gaze deviation, including the increasing hyperdeviation relative to the healthy viewing eye (circles connected with dashed lines) with adduction (Figs. 1A-C). On head tilt to the paretic side, the entire grid formed by the gaze directions of the covered paretic eye shifted upward relative to the viewing healthy eye and vice versa. When the healthy eye was covered, its pattern of vertical-horizontal gaze directions vertically mirrored the covered pathologic eye-that is, the hypodeviation relative to the viewing pathologic eye increased with abduction (Fig. 1D-F). On head tilt to the paretic side, the entire grid formed by the gaze directions of the covered healthy eye shifted downward.

To describe and model trajectories of the covered eye during dynamic Bielschowsky testing, when the viewing eye fixes on a specific target, two geometric locations must be known: (1) the starting position of the covered eye-that is, its position when the upright sitting patient monocularly fixes on a target with the other eye, and (2) the orientation of the axis about which the covered eye rotates during dynamic torsional vestibular stimulation.

Representative starting positions of covered eyes were obtained by pooling static eye positions of all patients. Figure 2 summarizes gaze directions associated with the nine fixation targets (hatched ellipses), when the left healthy (Fig. 2A) or the 
$35^{\circ}$ left ear down

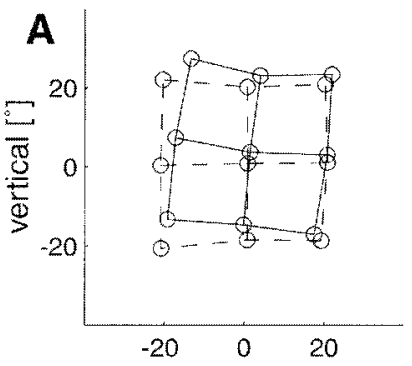

FiguRE 1. Example of static Bielschowsky head-tilt testing in right congenital trochlear nerve palsy (patient DS). Connected circles: stereographic projection of median horizontal and vertical gaze directions on the Hess screen. Solid lines: paretic right eye; dashed lines: unaffected left eye. (A-C) Paretic eye covered; (D-F) unaffected eye covered. (A, D) $35^{\circ}$ left-ear-down head position; (B, E) upright head position; $(\mathbf{C}, \mathbf{F}) 35^{\circ}$ right-ear-down head position.

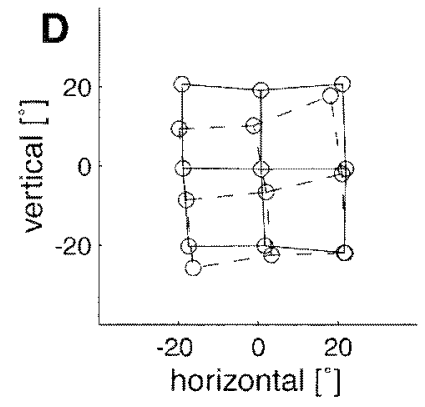

$35^{\circ}$ right ear down
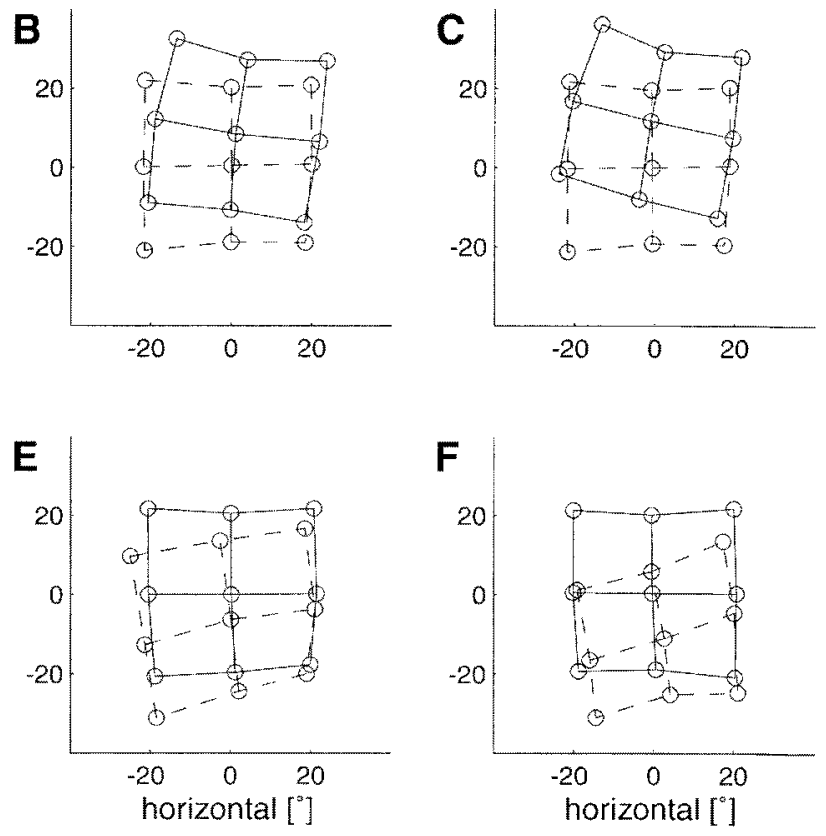

right paretic eye (Fig. 2B) was covered. Compared with healthy subjects (filled ellipses), average positions ( $\pm 1 \mathrm{SD}$ : horizontal and vertical radii of ellipses) of the covered paretic eyes in patients showed the typical pattern of increasing hyperdeviation in adduction, whereas the covered healthy eyes in patients showed the vertically mirrored pattern (see also Fig. 1).

The description of the ocular rotation axis during dynamic Bielschowsky head-tilt testing requires the computation of three-dimensional angular velocity vectors from eye positions, because these vectors describe the instantaneous rotation axes of the eyes in the head (see Appendix A). The panels in Figure 3 represent the top view ( $x-y$-plane) of angular velocity vectors of both eyes evoked by dynamic Bielschowsky head-tilt testing in the same patient whose static data is presented in Figure 1. Each panel contains populations of desaccaded angular velocity vectors of the right paretic (red dots) and the left healthy (blue dots) eye while the right eye was covered and the left eye was fixing on one of the nine target locations (subplots arranged according to the Hess screen scheme; for example, the left upper target corresponds to Fig. 3A). Throughout cycles of torsional turntable oscillation, angular velocity vec-

tors were scattered closely along a line in both the $x-y$ plane (shown) and $x-z$ plane (not shown). The axes of both eyes were not head fixed, but moved together with gaze direction. Nevertheless, the axes converged relative to each other in all gaze directions, and the vergence angle was larger than expected from the calculated convergence angle of $2.5^{\circ}$, when both ocular rotation axes would exactly point toward the target (distance: $1.4 \mathrm{~m}$ ). Also, the angles between the two axes increased somewhat with left gaze.

To determine the deviation of the ocular rotation axes from the lines of sight, which ideally should be zero to prevent horizontal-vertical retinal slip, ${ }^{3,6}$ the axes were stereographically projected on a Hess screen plot. Figure 4 illustrates the relation between ocular rotation axes and the respective gaze trajectories for the same patient whose data were depicted in Figures 1 and 3 . The rotation axes (asterisks connected with lines) of the viewing eye were always closer to the line of sight while the subject viewed the nine targets (crossings of dotted lines) than the rotation axes of the covered eye. The latter axes were displaced nasally, irrespective of whether the covered eye was the paretic one (Fig. 4B) or not (Fig. 4A). Thus, the

Figure 2. Gaze directions in upright position. Ellipses: average (center) $\pm 1 \mathrm{SD}$ (horizontal and vertical radius). Hatched: patients; filled: healthy subjects. (A) Gaze directions of left eyes (in patients: unaffected eyes) when covered. (B) Gaze directions of right eyes (in patients: paretic eyes) when covered. covered left eye

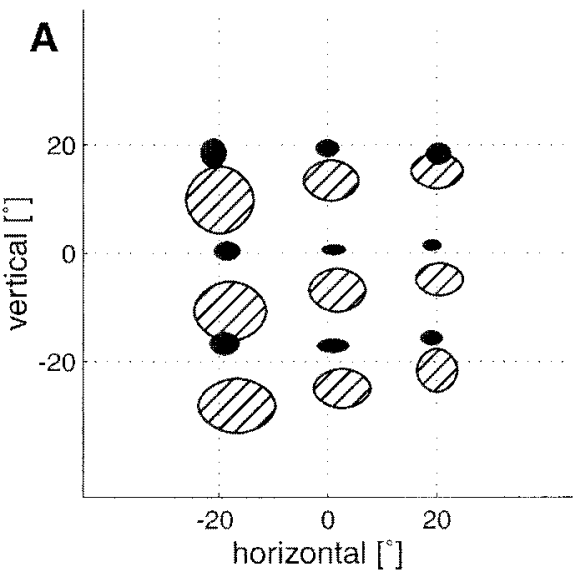

covered right eye

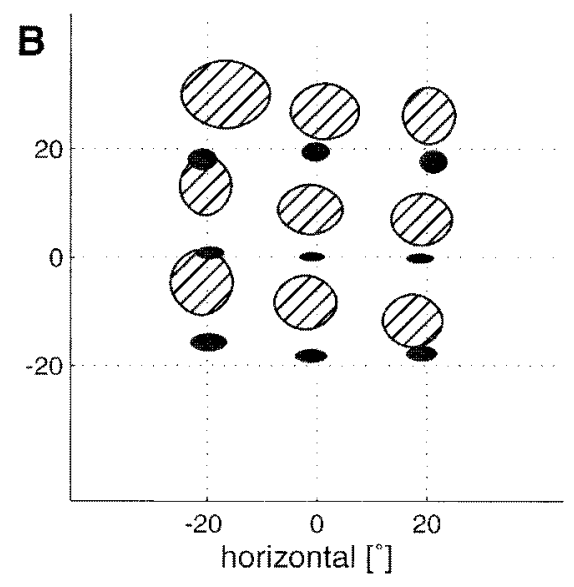



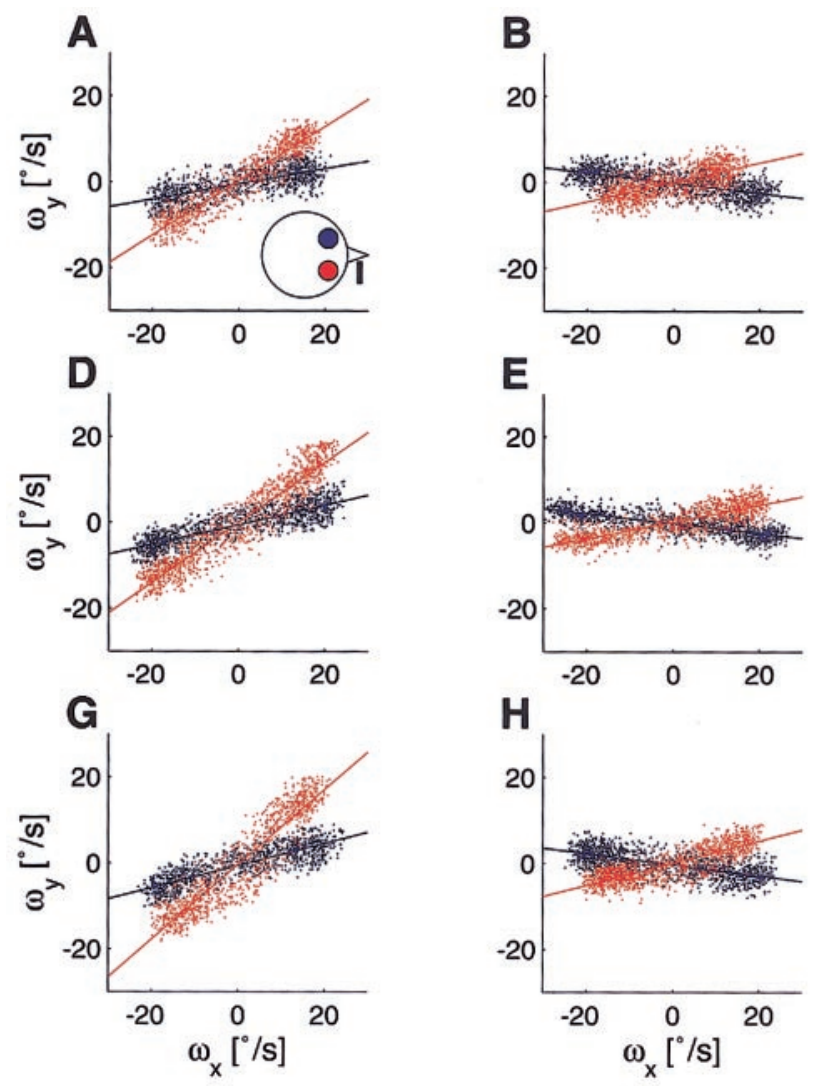

rotation axes of the two eyes always converged. Apart from the nasal shift, however, the ocular rotation axes of the covered eye maintained the relative configuration of the nine targets on the Hess screen. Even when the paretic eye was viewing, no obvious restrictions of rotation axes were evident.

The horizontal-vertical gaze trajectories that were produced by rotating the eyes about these axes were of low amplitude for the viewing eye and of higher amplitude when the eye was covered (Figs. 4C, 4D). Thus, the viewing eye closely kept the fixation of the target, because the rotation axis was near the line of sight, whereas the covered eye was not constrained by the visual target and rotated about a nasally displaced rotation axis. To better demonstrate the relation between rotation axes (Figs. 4A, 4B) and gaze directions (Figs. 4C, 4D), these plots were combined for the covered healthy (Fig. 4E) and paretic (Fig. 4F) eye (see videos, Example_Patient.mov and Hess_Screen.mov, Appendix B). Clearly, horizontal-vertical gaze trajectories of both covered eyes were a result of rotating about the axes in a circular fashion, when positions (gaze directions) and axes were stereographically projected. Note again that the rotation axes deviated from the line of sight in the nasal direction in both the covered healthy and the covered palsied eyes. In addition to this horizontal deviation, there was a more variable vertical deviation between the gaze direction and the location of the rotation axis. On average, rotation axes of the paretic right eye were below its hyperdeviated gaze directions, and rotation axes of the healthy eye were above its hypodeviated gaze directions. Exceptions were the adducted positions of the covered healthy eye.

Figure 5 summarizes the locations of the rotation axes during monocular fixation of the nine cardinal gaze directions in patients and healthy subjects. The paretic right eyes, when covered (Fig. 5B, hatched ellipses), showed rotation axes that, on average, were shifted nasally compared to the control subjects (filled ellipses). This horizontal deviation increased with

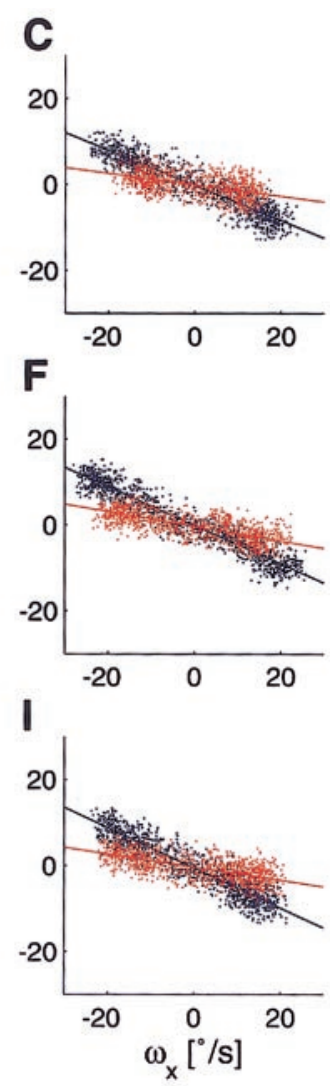

Figure 3. Example of angular velocity vectors in right-side congenital trochlear nerve palsy during dynamic Bielschowsky head-tilt testing (patient DS). Graphs represent the top projection ( $x-y$-plane) of desaccaded velocity traces. Angular velocity vectors of the covered paretic right eye are indicated by red dots, and those of the viewing healthy left eye by blue dots. Lines: first-order linear regressions. Panels are arranged according to the nine targets of the Hess screen scheme (e.g., the top left target corresponds to $\mathbf{A})$.

adduction of the covered paretic eye. Compared to healthy subjects, the horizontal and vertical standard deviations $( \pm 1$ $\mathrm{SD}$; horizontal and vertical radii of ellipses) of rotation axes for the different gaze directions were larger in patients. The healthy left eyes of patients, when covered (Fig. 5C, hatched ellipses), showed a nasal shift of the rotation axes as well, but in contrast to the paretic eye, the deviation increased with abduction, not adduction. The vertical deviation of axes of the covered eyes in patients was small.

The rotation axes of healthy left eyes in patients and left eyes in healthy subjects matched closely in the viewing condition (Fig. 5A), and the rotation axes in both groups of subjects were relatively near the line of sight when fixation was maintained by the respective eye. Also, the rotation axes of the paretic eyes, when viewing, were kept close to the gaze direction-that is, showed only a minor nasal deviation compared to the control subjects (Fig. 5D). The eyes of the healthy subjects (filled ellipses) had their rotation axes close to the line-of-sight pointing to the nine target directions, irrespective of whether the eyes were viewing or not, but a small nasal and downward deviation was still visible, especially during temporal- upward fixation.

In the patients, Figure 6 demonstrates the stability of ocular rotation axes of viewing healthy (Fig. 6A) and covered paretic (Fig. 6B) eyes during dynamic Bielschowsky head-tilt testing. The average ocular rotation axes (centers of ellipses) are identical with the previous figure, but the horizontal and vertical ranges were determined by pooling the horizontal and vertical deviations of axis data from their average values over all patients. The orientation of small angular velocity vectors, which occur when chair velocity crosses zero, is unstable. Therefore, for this plot, we eliminated the $20 \%$ shortest angular velocity vectors before computing the horizontal and vertical standard deviations of their stereographic projection on the Hess screen. Both the viewing healthy and the covered paretic eyes 

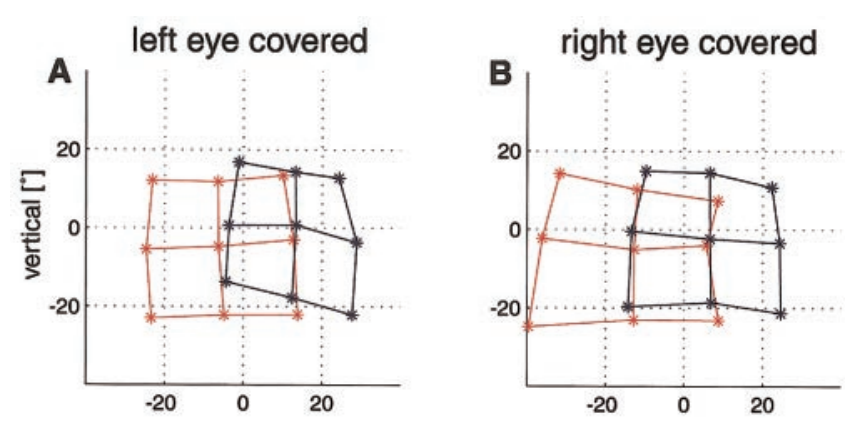

lated static positions of the covered paretic eye in a patient sitting upright by rotating the nine gaze directions of a fixating healthy eye about a rotation axis determined by the pulling direction of the superior oblique muscle $\mathbf{m}_{\text {so }}=(-0.80,0.57$, $-0.17) .{ }^{18,19}$ In our example, we rotated by $15^{\circ}$. This resulted in a vertical deviation of $8.8^{\circ}$ with the healthy eye fixating straight ahead. This vertical deviation is equal to the mean deviation measured in our patients $\left(8.8 \pm 4.6^{\circ}[\mathrm{SD}]\right)$; thus, the rotation by $15^{\circ}$ appears to be realistic. The resultant pattern of the nine simulated eye positions (Fig. 7B) closely resembles the actual data shown in Figure 1B (typical example) and Figure 2B (summary plot).

In a second step, the positions of the covered paretic eye
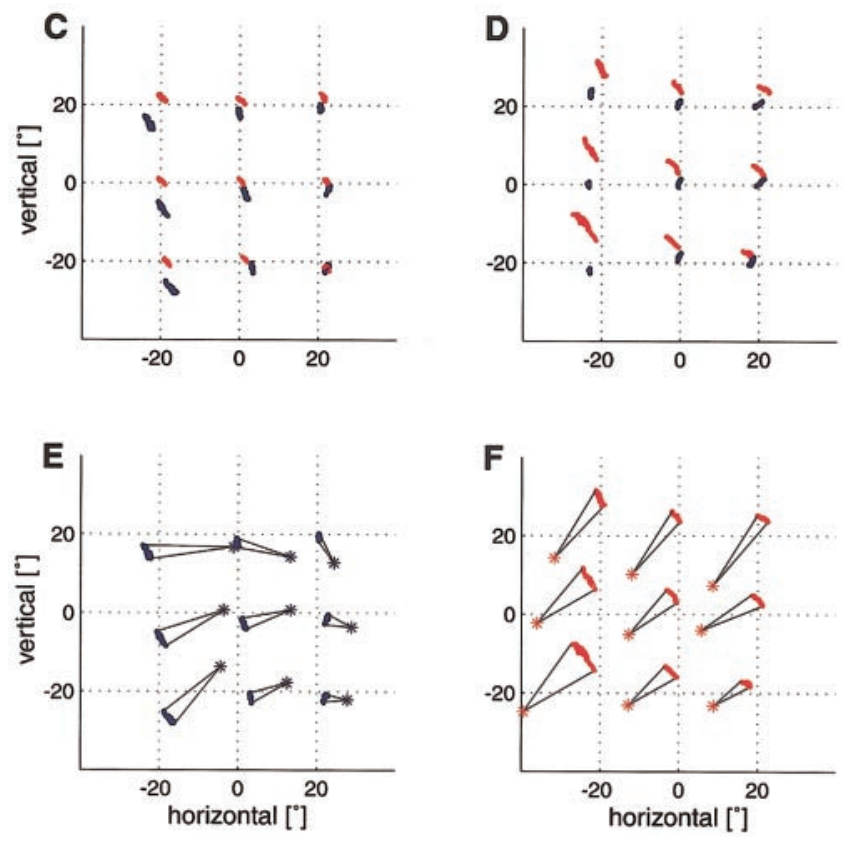

FIGURE 4. Example of projections of ocular rotation axes (*) and gaze trajectories (thick lines) on the Hess screen (patient DS). Red: paretic right eye; blue: unaffected left eye. Intersections of dotted lines: location of visual targets. (A, B) Ocular rotation axes; (C, D) gaze trajectories; (E, F) combined plot of ocular rotation axes and gaze trajectories (thin lines: sectors of circular gaze trajectories around ocular rotation axes).

showed similar horizontal and vertical stability of rotation axes. The range in the covered paretic eye, however, was somewhat larger than in the viewing healthy eye.

\section{Computer Simulations}

The trajectories of covered paretic and healthy eyes during dynamic Bielschowsky head-tilt testing in patients with trochlear nerve palsy can be explained by a few geometric assumptions. Although detailed biomechanical models of the eye plant, such as Orbit ${ }^{16}$ or the computerized strabismus model, ${ }^{17}$ have the advantage of being anatomically more realistic, a simple geometric model makes it easier to understand, why the dynamic Bielschowsky head-tilt test evokes the eye movements that we observed. The geometric model presented here computes rotation vectors, but, for convenience, the results are projected stereographically on the Hess screen chart. It must be stressed that this is a purely qualitative model with the least assumptions possible (e.g., it does not take into account the effects of eye positions on muscle-pulling directions).

Figure 7 demonstrates the geometric two-step model of the dynamic Bielschowsky head-tilt test. In a first step, we simu-

were rotated by $\pm 10^{\circ}$ about a rotation axis that deviated $15^{\circ}$ nasally from the axis of the viewing healthy eye (Fig. 7D). The chosen amplitude $\left(10^{\circ}\right)$ of torsional eye movements during roll rotation is close to the average value in our patients $(7.9 \pm$ $\left.2.3^{\circ}\right)$. Similarly, the nasal deviation of the rotation axis $\left(15^{\circ}\right)$ was also chosen to be near to the average value measured in the patients $\left(17.1 \pm 5.8^{\circ}\right)$. Owing to the properties of stereographic projection of our Hess screen scheme, gaze trajectories describe circular arcs about the fixed rotation axes (Figs. $7 \mathrm{C}_{1}$, $\left.7 C_{2}, 7 D\right)$. Even by keeping the horizontal axis deviation constant for every gaze direction and without adding an additional vertical component, the model closely reproduced the gaze trajectories of the patient in the example (Fig. 4F; see video, Geometric_Model.mov, Appendix B).

To simulate the dynamic Bielschowsky test with the healthy (left) eye covered, we first assumed that adaptively increased innervation of the paretic SO leads to a relative hyperinnervation of the IR of the healthy eye (yoke muscle). We therefore simulated left IR overaction by rotating the nine static gaze directions of a fixating eye by $15^{\circ}$ about the unit vector $\mathbf{m}_{\mathrm{IR}}=$ $(-0.41,0.86,0.32)^{18,19}$ (Fig. $\left.7 A_{1}\right)$. The pattern of gaze trajectories during rotation $\pm 10^{\circ}$ about an axis that was again $15^{\circ}$ nasally displaced (Fig. $7 \mathrm{C}_{1}$ ) did not satisfactorily reproduce the trajectories seen in most of the patients (Fig. 4E), because the hypodeviation along horizontal eye positions did not vary.

We therefore implemented Hering's law, not on a muscleby-muscle basis (ipsilateral SO by contralateral IR), but rather let the covered healthy eye be hyperinnervated so that it was rotated about an axis parallel to the axis of the SO of the paretic eye, but in the opposite direction. We called this mechanism "yoke hyperinnervation" (Fig. $7 \mathrm{~A}_{2}$ ). Dynamic $\pm 10^{\circ}$ rotation about an axis that was $15^{\circ}$ nasally displaced from the line of sight of the viewing eye led to gaze trajectories (Fig. $7 \mathrm{C}_{2}$ ) that matched the actual data better (Fig. 4E), as the hypodeviation of the covered healthy eye decreased with adduction.

\section{Discussion}

To elucidate the mechanism of the positive Bielschowsky headtilt sign, we determined the orientation of ocular rotation axes of both eyes during dynamic roll oscillation in patients with unilateral trochlear nerve palsy. Although the axes of viewing paretic or healthy eyes remained close to the line of sight to prevent retinal slip, the axes of covered paretic or healthy eyes always pointed nasally relative to the line of sight. In all gaze directions, ocular rotation axes of viewing and covered eyes were relatively stable over an entire cycle of torsional vestibular stimulation. A simple geometric model that simulated (1) a static deviation of the covered paretic eye opposite to the pulling direction of the palsied superior oblique muscle or a static deviation of the covered healthy eye along this direction, and (2) a subsequent dynamic oscillation about a nasally deviated rotation axis, closely reproduced the experimental data found for the covered eyes. 

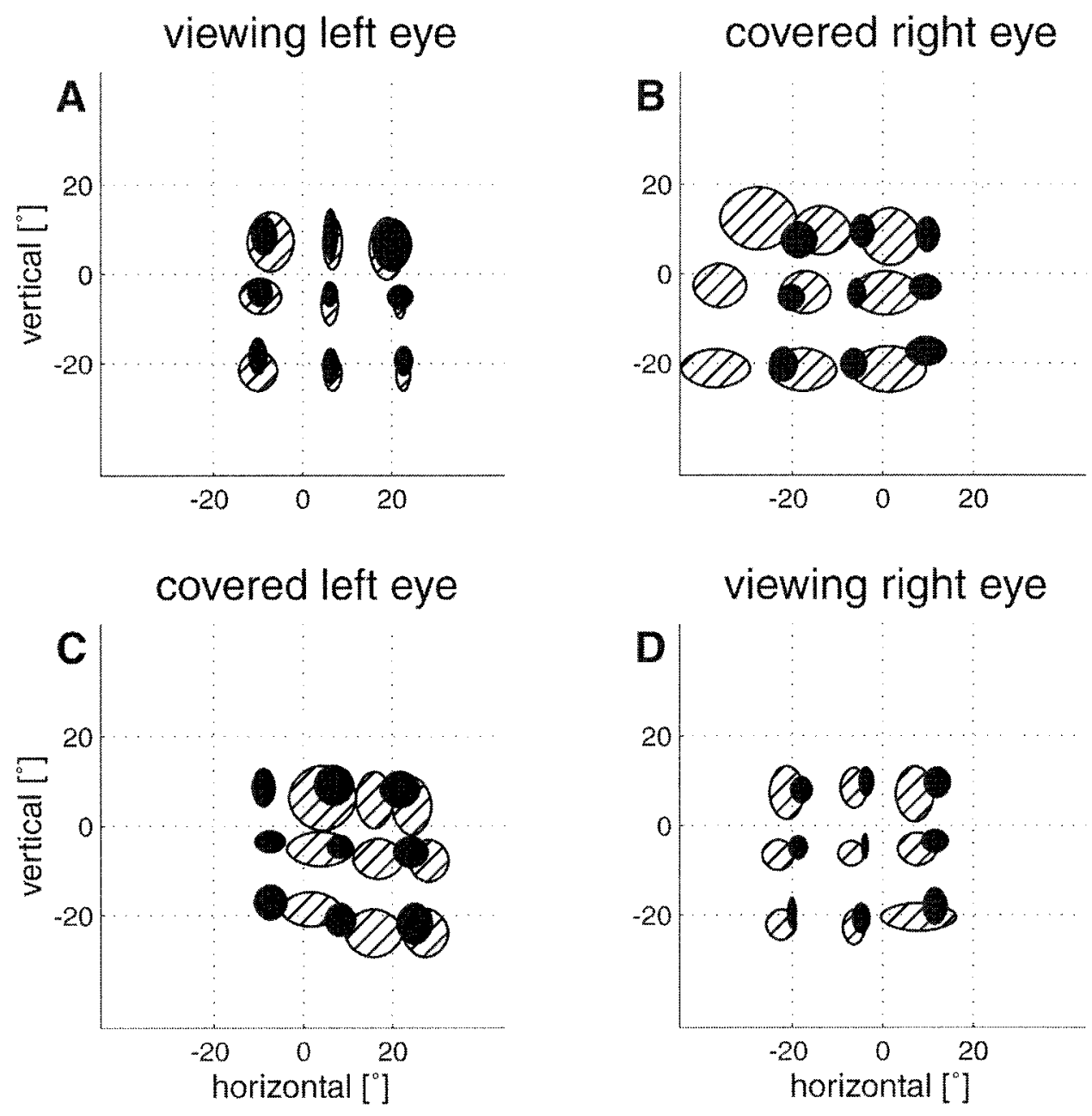

FiguRE 5. Orientation of ocular rotation axes. Ellipses: average (center) $\pm 1 \mathrm{SD}$ (horizontal and vertical radius). Hatched: patients; filled: healthy subjects. Intersections of dotted lines: location of visual targets. (A, B) Left eye viewing condition; (C, D) right eye viewing condition.

\section{Orientation of Ocular Rotation Axes}

Pathologic gaze trajectories during torsional vestibular stimulation can best be understood if we consider the orientation of rotation axes of both eyes relative to the line of sight. Only if an ocular rotation axis stays aligned with the line of sight, will the line of sight not move during vestibular stimulation. Deviations between the line of sight and the ocular rotation axis lead on the Hess screen to circular movements of the line of sight about the ocular rotation axis during vestibular stimulation. ${ }^{3,6}$ In the case of a nasal deviation of the ocular rotation axis from the line of sight, for instance, an extorsional eye movement moves the line of sight downward.

Although the deviation between the ocular rotation axis and the line of sight can explain the horizontal-vertical trajectories of single eyes, the relative orientation between the ocular rotation axes of both eyes must also be considered. Only if the angle between these axes equals the computed convergence angle for binocular fixation of targets at certain distances, will the lines of

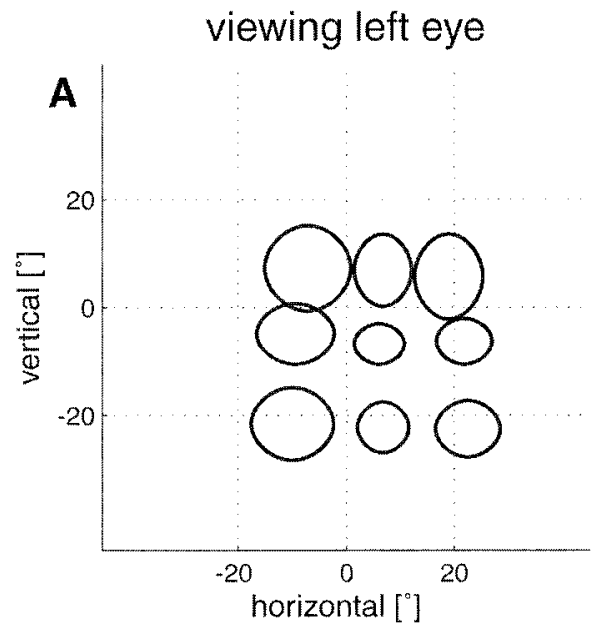

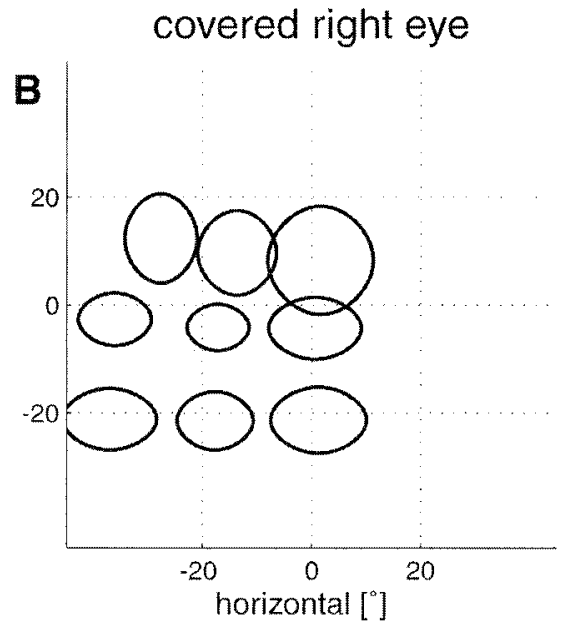

FIGURE 6. Stability of ocular rotation axes during dynamic Bielschowsky head-tilt testing. Pooled data of all patients after discarding the $20 \%$ shortest angular velocity vectors. Center of ellipses: average location. Radius of ellipses: average horizontal and vertical standard deviation. (A) unaffected (left) eye; (B) paretic (right) eye. 
FigURE 7. Geometric two-step model to simulate gaze trajectories evoked by dynamic Bielschowsky head-tilt testing. Projection of gaze direction and ocular rotation axes on the Hess screen. ( $\left.\mathbf{A}_{1}, \mathbf{A}_{\mathbf{2}}, \mathbf{B}\right)$ First step of simulation. Static gaze directions in upright position. Circles connected by solid lines: paretic right eye; circles connected by dashed lines: healthy left eye. $\left(\mathbf{C}_{1}, \mathbf{C}_{2}, \mathbf{D}\right)$ Second step of simulation. Rotation about nasally deviated constant ocular rotation axes (*) leads to circular gaze trajectories (thick lines). Intersections of dotted lines: locations of visual targets. $\left(\mathbf{A}_{\mathbf{1}}, \mathbf{C}_{\mathbf{1}}\right)$ Left-side hyperinnervation of the IR; $\left(\mathbf{A}_{2}, \mathbf{C}_{2}\right)$ left-side hyperinnervation along a vector parallel to the vector of the $\mathrm{SO}$ on the right side (yoke vector); (B, D) right-sided paresis of SO
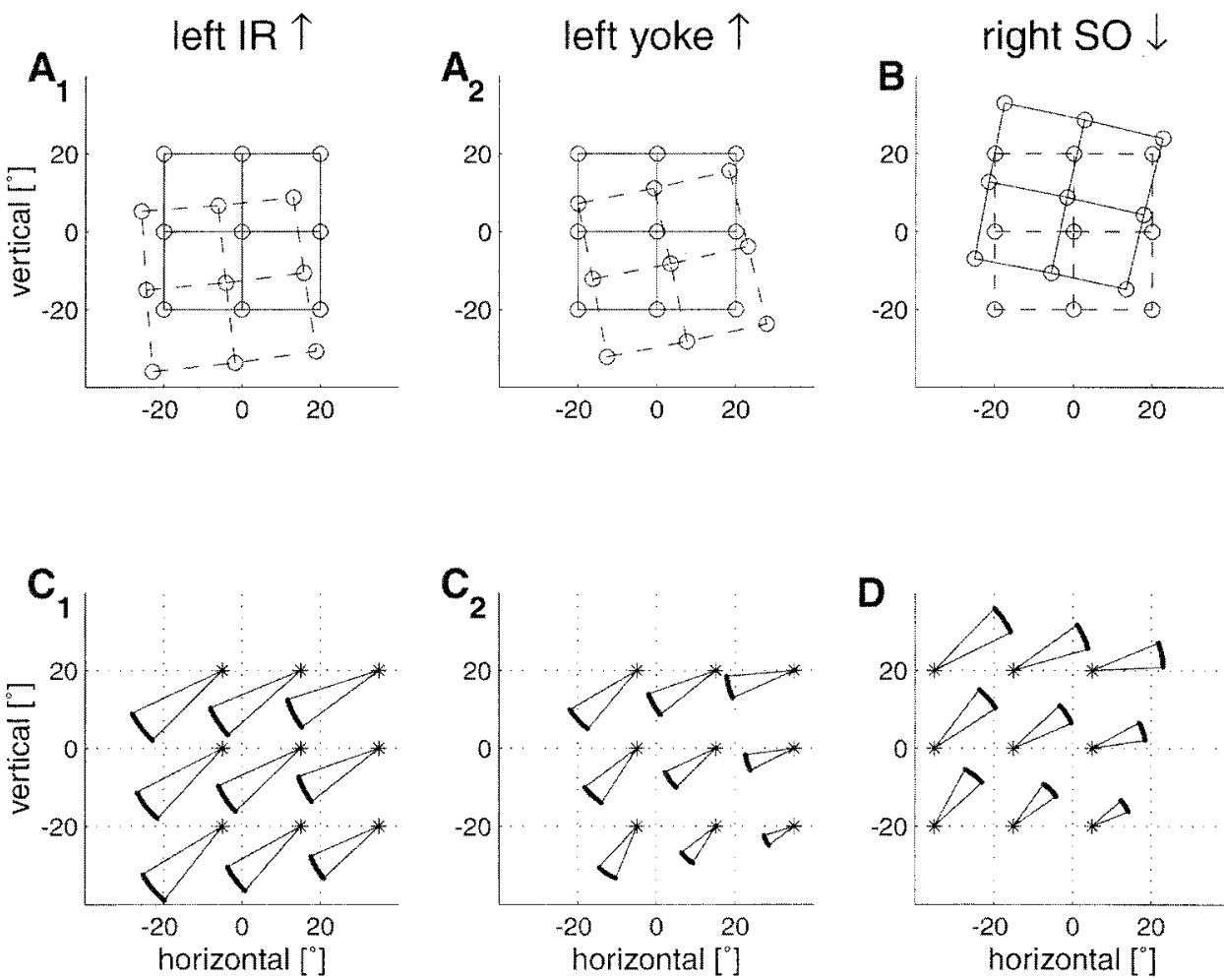

sight of both eyes not deviate during vestibular stimulation. ${ }^{6}$ For example, exaggerated convergence of rotation axes makes the line of sight of the intorting eye move upward and the line of sight of the extorting eye move downward relative to the fellow eye.

Because a small nasal and downward deviation of the ocular rotation axis from the line of sight can be seen even in healthy subjects (Fig. 5), the appearance of small disconjugate vertical (due to convergence of rotation axes) and conjugate horizontal (due to downward deviation of rotation axes) eye movements during torsional vestibular stimulation represents a normal finding. In fact, these disconjugate vertical ${ }^{20,21}$ and conjugate horizontal $^{20}$ eye movements were already noted in previous studies.

\section{Nasal Deviation of Rotation Axes of Covered Eyes in Trochlear Nerve Palsy}

Figure 8 schematically explains why in patients with trochlear nerve palsy the ocular rotation axis of the covered paretic or

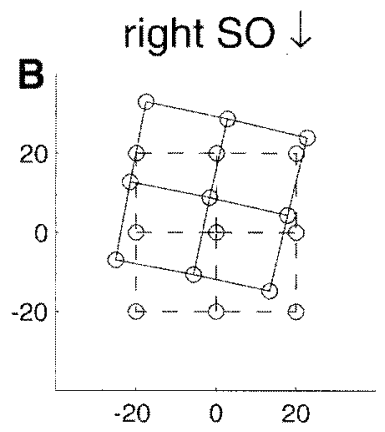

covered healthy eye is rotated nasally and why the amount of this nasal rotation depends on horizontal gaze direction. Our qualitative explanation is based on the assumption that an isolated contraction of one extraocular muscle rotates the eye about an axis perpendicular to the pulling direction of this muscle. The rotation axis of an individual muscle and its impact on the overall rotation of the eye can be described by an angular velocity vector. The total angular velocity vector during synergistic activity of all contributing extraocular muscles is then determined by the vectorial addition of all corresponding angular velocity vectors, which is equivalent to a scheme described by Hering, who used the term Halbaxen (German term for half-axes) for similar vectors. ${ }^{5}$ The vectorial addition of angular velocity vectors implies that within the sector of eye movements associated with contraction of a particular muscle, paresis of this muscle tilts the resultant ocular rotation axis away from the axis of the palsied muscle, and muscle overac-
FiguRE 8. Geometric explanation of why, in trochlear nerve palsy, rotation axes of both eyes are nasally displaced when covered. All sketches present a top view. (A-C) unaffected left eye; (D-F) paretic right eye. a: angular velocity vector along the line of sight. SO $\downarrow$ : angular velocity vector representing the deficit of the SO. $\mathbf{Y}_{\text {SO }} \uparrow:$ angular velocity vector (yoke vector) representing the hyperinnervation parallel to the vector of the contralateral SO. b: resultant angular velocity vector.

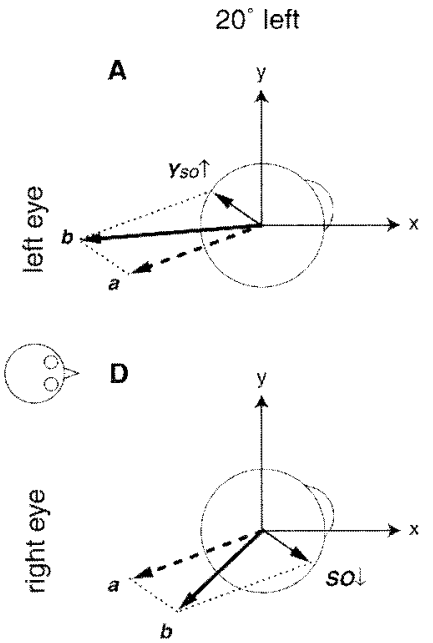

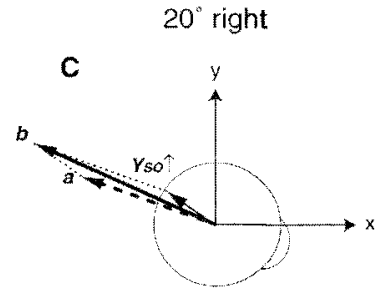

E
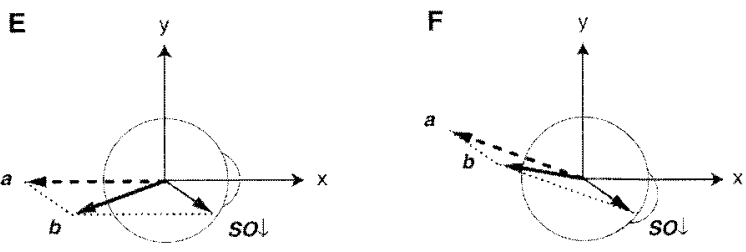
tion pulls the resultant ocular rotation axis toward the axis of the overacting muscle.

When the covered paretic right eye points straight ahead, the line of sight aligns with the $x$-axis of the coordinate system (Fig. 8E). Let us assume that the head rotates right-ear-down about an axis that is parallel to the $x$-axis. Driven by the vestibulo-ocular reflex, the right eye makes a compensatory movement in the intorsional direction. In healthy subjects, the elicited angular velocity vector $\mathbf{a}$, resulting from transient force changes in all six extraocular muscles, is parallel to the line of sight (negatively directed according to the right-hand rule). In superior oblique muscle paresis, the angular velocity vector in the direction of this muscle is lacking. Thus, an angular velocity vector SO $\downarrow$ pointing into the opposite direction of the superior oblique muscle vector $\left(35.5^{\circ}\right.$ abduction) must be added to vector $\mathbf{a}$. The resultant angular velocity vector $\mathbf{b}$ represents an ocular rotation axis that is deviated nasally.

Because in healthy subjects the ocular rotation axis tends to be aligned with the line of sight, even when the eye is covered, ${ }^{6}$ but the pulling direction of the superior oblique muscle is approximately head-fixed when gaze is changed in the horizontal direction, ${ }^{19}$ the angle between angular velocity vectors a and SO $\downarrow$ increases in abduction (Fig. 8F) and decreases in adduction (Fig. 8D). As a consequence, the deviation of the resultant angular velocity vector $\mathbf{b}$ from the line of sight is largest in adduction, where vectors a and SO $\downarrow$ are almost orthogonal, and smallest in abduction, where vectors $\mathbf{a}$ and SO $\downarrow$ are almost opposed. This pattern of increasing deviation of ocular rotation axes from the line of sight with gaze toward the unaffected eye agrees well with our data of covered paretic eyes in patients (Fig. 5B).

Based on Hering's law, we postulate a hyperinnervation of the covered unaffected eye along a vector in the direction of the vector representing the contralateral paretic superior oblique muscle. We shall call that vector "yoke vector of the contralateral superior oblique muscle." Contralateral superior oblique muscle palsy leads to hyperinnervation along this vector $\left(\mathbf{Y}_{\text {SO }} \uparrow\right)$. Thus, $\mathbf{Y}_{\text {SO }} \uparrow$ is added to $\mathbf{a}$, and the resultant rotation axis $\mathbf{b}$ also deviates nasally from the line of sight (Fig. $8 \mathrm{~B})$. As in the right paretic eye, deviation of the rotation axis from the line of sight increases with gaze toward the unaffected eye. This deviation pattern of the ocular rotation axis from the line of sight in the covered healthy eye also corresponds well with the one observed in patients (compare Fig. 5C).

Our hypothesis explaining the eye-position dependence of the deviation between the ocular rotation axis and line of sight during dynamic Bielschowsky head-tilt testing applies only to the horizontal direction. In the vertical direction, the pulling direction of the superior oblique muscle changes with vertical gaze direction, ${ }^{19}$ because the point of insertion behind the equator in the superior-temporal quadrant of the eyeball moves anteriorly when the eye points downward and posteriorly when the eye points upward. Therefore, the deviation between ocular rotation axis and line of sight should change much less with vertical gaze direction, which is confirmed by our data (compare Figs. 5B, 5C).

\section{Hering's Law}

Strabismus due to palsy of a single muscle in one eye is always disconjugate-that is, the deviation between the two eyes increases when gaze is moved in the pulling direction of the paretic muscle. Because of Hering's law of equal innervation, ${ }^{5}$ there is an unambiguous relationship between the positions of the two eyes, independent of whether the paretic or the healthy eye is covered. ${ }^{22}$ This rule can best be explained with an example of a patient with right-side abducens nerve palsy.
If this patient fixes with his healthy left eye on a target $10^{\circ}$ to the left, the covered paretic right eye points $15^{\circ}$ to the left (squint angle: $5^{\circ}$ ). The two eyes will keep the same position, if the healthy left eye is covered and the paretic right eye now fixes on a target $15^{\circ}$ to the left (squint angle: $5^{\circ}$ ). If, however, the paretic right eye is forced to fix on the target $10^{\circ}$ to the left, the left healthy eye points straight ahead (squint angle: $10^{\circ}$ ). In other words, Hering's law of equal innervation leads to different squint angles, depending on whether the healthy or the paretic eye is fixing on the very same target. For a given target, viewing with the healthy eye (primary deviation) produces a smaller squint angle than viewing with the paretic eye (secondary deviation). ${ }^{23}$ This rule applies to paretic strabismus in general, including trochlear nerve palsy. Our data reveal that Hering's law has similar consequences on the orientation of ocular rotation axes during dynamic Bielschowsky head-tilt testing. While the rotation axis of both the paretic and healthy eye moves nasally in the covered condition, its deviation from the rotation axis of the viewing eye increases in the direction in which the paretic eye adducts (Figs. 8A, 8D).

To test whether the eye-position- dependent angle between the rotation axes of both eyes follows the same principle as the incomitant squint angle, we used the graphic method to analyze strabismus proposed by Zee et al. ${ }^{22}$ but plotted the absolute horizontal angle of ocular rotation axes of both eyes, instead of their horizontal gaze position, against each other (Fig. 9A). Data points of axes moving in parallel (i.e., comitant) would lie on a line with zero intercept and a slope of 1 (dashed line). Data points below this line represent convergent axes, and data points above the line represent divergent axes. A slope of 1 indicates that axes are comitant; otherwise they are incomitant. Data points of healthy subjects with either eye covered lay on a regression line with a slope of almost 1 , indicating comitance (squares: average of pooled data from fixations along the horizontal meridian; filled squares: right eye covered; open squares: left eye covered). Thus, analogous to binocular eye positions, binocular axes were comitant in healthy subjects, indicating that Hering's law of equal innervation extends to the orientation of rotation axes. Consistent with the fact that axes normally show a slight convergence (compare with Fig. 5), data points were situated below the dashed line.

The slopes of regression lines in patients (triangles: average of pooled data from fixations along the horizontal meridian) were larger than 1, demonstrating incomitance between the rotation axes of both eyes. As in healthy subjects, the fitted lines through the data with either eye covered (Fig. 9, filled: right eye covered is "primary axis deviation"; open: left eye covered is "secondary axis deviation") were similar and therefore, for a given axis orientation of one eye, the angle between the ocular rotation axes was almost the same, independent of which eye was covered. Note, however, that, for a given orientation of an ocular rotation axis in one eye, the deviation of the axis of the fellow eye was larger when the former eye was the healthy one. Thus, the "primary axis deviation" was larger than the "secondary axis deviation." This is exactly opposite to what one observes for eye positions with the primary squint angle being smaller than the secondary squint angle.

The two lines intersected the normal regression line at approximately $40^{\circ}$ abduction of the rotation axis of the paretic right eye (filled arrowhead: right eye covered; open arrowhead: left eye covered). In fact, At $35.5^{\circ}$ right gaze, rotation axes are roughly parallel to the SO $\downarrow$ (covered palsied eye) and the $\mathbf{Y}_{\text {so }} \uparrow$ vector (covered healthy eye). At this orientation, neither vector has an impact on the orientation of the resultant angular velocity vector. Here, SO $\downarrow$ and $\mathbf{Y}_{\text {SO }} \uparrow$ only influence the length of the resultant angular velocity vector. 

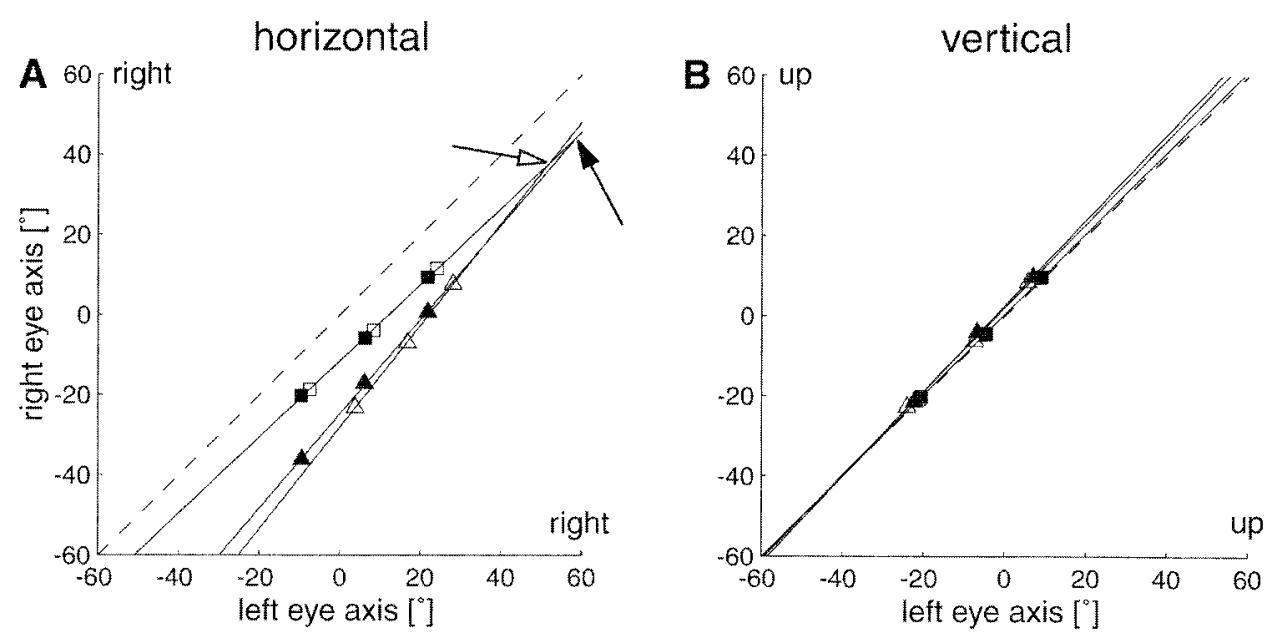

FIGURE 9. Comitance and vergence of ocular rotation axes. Orientations of rotation axes of both eyes plotted against each other. Triangles: average data in patients; squares: average data in healthy subjects; open symbols: right eye viewing condition; filled symbols: left eye viewing condition. Dashed line: absolute comitance and no vergence between both eyes. Solid lines: first-order linear regression. Data points below dashed line: convergence. Slope $>1$ : increasing convergence of axes with left gaze. Arrows: intersection of regression line from healthy subjects with regression lines from patients. Open arrow: right eye viewing condition; filled arrow: left eye viewing condition. (A) Horizontal components; fixations along horizontal meridian; (B) vertical components; fixations along vertical meridian.

For fixations along the vertical meridian (Fig. 9B), axis orientations in patients and healthy subjects coincided and scattered along the dashed line indicating parallel orientation and comitance.

\section{Computer Simulation with Biomechanical Plant Model}

We have explained the nasal displacement of the ocular rotation axis during dynamic Bielschowsky head-tilt testing in superior oblique muscle palsy by simply subtracting the angular velocity vector of this muscle from the global angular eye velocity vector. To test whether a biomechanical model of the eye plant would, for the covered eye, also predict the nasal deviation of the ocular rotation axis and its stability during vestibular stimulation, we used EyeLab, a MatLab implementation $^{15}$ of the Orbit model. ${ }^{16}$ We simulated sinusoidal eye rotations with an amplitude of $\pm 10^{\circ}$ about the line of sight at the same nine gaze directions as in our experiment. All active and passive forces of the superior oblique muscle were set to zero, and the resultant gaze directions were plotted on the Hess scheme, as shown in Figure 10. Similar to the gaze trajectories recorded in patients (Fig. 4F), the vertical deviation of eye position from the targets increased when the head was tilted to the affected side and decreased when the head was tilted to the healthy side (Fig. 10A). EyeLab correctly predicted the nasal displacement of ocular rotation axes (Fig. 10B), but their orientations (solid lines) vastly oscillated around the initial location in upright position (asterisks). Thus, although EyeLab was able to predict the vertical deviation of the covered eye qualitatively, it failed to hold its rotation axis stable during torsional stimulation (compare with Fig. 6; see video, Eyelab_Model. mov, Appendix B).

The EyeLab model used a biomechanical approach, trying to simulate the experiment with a universal model of the eye plant. The two-step model, however, intended to reproduce the eye movements measured in our experiment with a geo-
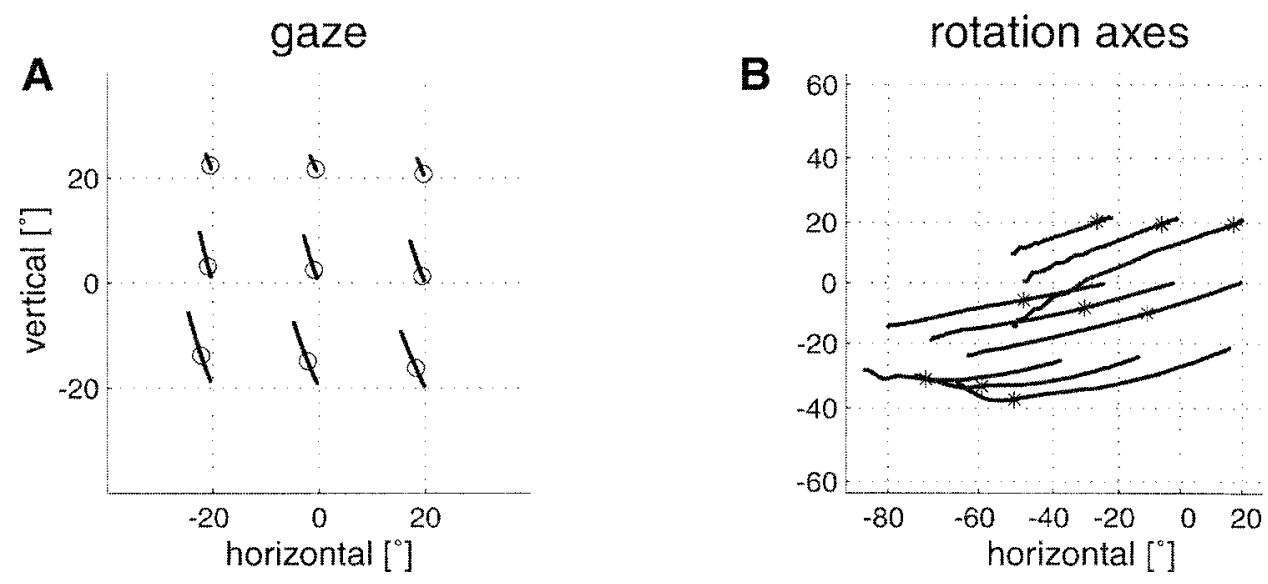

FIGURE 10. Computer simulation of right superior oblique muscle palsy during dynamic Bielschowsky head-tilt testing. Projection of gaze and rotation axis orientation on Hess screen. (A) Gaze trajectories. Open circles: gaze in upright, whole-body position; (B) trajectories of axes orientations. (*) Axes orientations in upright, whole-body position. 
metric approach, using the fewest assumptions possible. Comparing the relative accuracy of the two models, the two-step model holds for our paradigm and elucidates the underlying axis deviations, but allows only qualitative statements about the ocular kinematics in trochlear nerve palsy. The EyeLab model on the other hand, attempts to be anatomically realistic. It was able to predict the vertical deviation of the covered eye qualitatively, but it failed to hold its rotation axis stable. This failure to reproduce the experimental data could be due to compensatory changes in other eye muscles. Because little is known about the biomechanical changes of individual muscles in chronic trochlear nerve palsy, we did not simulate more complicated scenarios.

\section{Conclusion}

Based on three-dimensional eye movement recordings in patients with unilateral trochlear nerve palsy, we provided a kinematic explanation of the Bielschowsky head-tilt sign. The pattern of incomitance of ocular rotation axes suggests that Hering's law of equal innervation has not only positional, but also kinematical consequences on eye rotation in patients with eye muscle palsies.

\section{Acknowledgments}

The authors thank Chris Bockisch and Albert Züger for technical support and Tanja Schmückle for assistance during the experiments.

\section{References}

1. Schmid-Priscoveanu A, Straumann D, Kori AA. Torsional vestibuloocular reflex during whole-body oscillation in the upright and the supine position. I. Responses in healthy human subjects. Exp Brain Res. 2000;134:212-219.

2. Groen E, Bos JE, de Graaf B. Contribution of the otoliths to the human torsional vestibulo-ocular reflex. J Vestib Res. 1999;9:27-36.

3. Misslisch $\mathrm{H}$, Tweed $\mathrm{D}$, Fetter $\mathrm{M}$, et al. Interaction of smooth pursuit and the vestibuloocular reflex in three dimensions. $J$ Neurophysiol. 1996;75:2520-2532.

4. Misslisch H, Tweed D, Fetter M, et al. Rotational kinematics of the human vestibuloocular reflex III. Listing's Law. J Neurophysiol. 1994;72:2490-2502.

5. Hering E. Die Lehre vom binocularen Sehen. Leipzig, Germany: Verlag von Wilhelm Engelmann; 1868

6. Bergamin O, Straumann D. Three-dimensional binocular kinematics of torsional vestibular nystagmus during convergence on headfixed targets in humans. J Neurophysiol. 2001;86:113-122.

7. Bielschowsky A, Hofmann FB. Die Verwerthung der Kopfneigung zur Diagnostik von Augenmuskellähmungen aus der Heber- und Senkergruppe. The evaluation of head inclination for the diagnosis of pareses of ocular muscles from the group of elevators and depressors (in German). Graefes Arch Opth. 1900;51:174-185.

8. van Rijn LJ, Van der SJ, Collewijn H. Instability of ocular torsion during fixation: cyclovergence is more stable than cycloversion. Vision Res. 1994;34:1077-1087.

9. Hess WR. Eine neue Untersuchungsmethode bei Doppelbildern. Arch Augenhk. 1909;62:233-238.

10. Parks MM. Isolated cyclovertical muscle palsy. Arch Ophthalmol. 1958;60:1027-1035.

11. Bergamin O, Zee DS, Roberts DC, et al. Three-dimensional Hess screen test with binocular dual search coils in a three-field magnetic system. Invest Ophthalmol Vis Sci. 2001;42:660-667.

12. Straumann D, Zee DS, Solomon D, et al. Transient torsion during and after saccades. Vision Res. 1995;35:3321-3334.
13. Haustein W. Considerations on Listing's Law and the primary position by means of a matrix description of eye position control. Biol Cybern. 1989;60:411-420.

14. Hepp K. On Listing's law. Commun Math Phys. 1990;132:285-292.

15. Porrill J, Warren PA, Dean P. A simple control law generates Listing's positions in a detailed model of the extraocular muscle system. Vision Res. 2000;40:3743-3758.

16. Miller JM, Shamaeva I, Pavlovski DS. Orbit ${ }^{T M}$ Gaze Mechanics Simulation (Version 1.5). San Francisco: Eidactics; 1995.

17. Simonsz HJ, Spekreijse H. Robinson's computerized strabismus model comes of age. Strabismus. 1996;4:31- 46.

18. Volkmann AW. Zur Mechanik der Augenmuskeln. Ber Verb Sachs Ges Wsch. 1869;21:28-69.

19. Robinson DA. A quantitative analysis of extraocular muscle cooperation and squint. Invest Ophthalmol. 1975;14:801-825.

20. Jauregui-Renaud K, Faldon ME, Gresty MA, et al. Horizontal ocular vergence and the three-dimensional response to whole-body roll motion. Exp Brain Res. 2001;136:79-92.

21. Kori AA, Schmid-Priscoveanu A, Straumann D. Vertical divergence and counterroll eye movements evoked by whole-body position steps about the roll axis of the head in humans. J Neurophysiol. 2001;85:671-678.

22. Zee DS, Chu FC, Optican LM, et al. Graphic analysis of paralytic strabismus with the Lancaster red-green test. Am J Ophthalmol. 1984;97:587-592.

23. von Noorden GK, Campos EC. Binocular Vision and Ocular Motility. 6th ed. St. Louis: Mosby; 2002:415-416.

\section{Appendix A}

A rotation vector $\mathbf{r}^{\mathbf{e}}\left(r_{\mathrm{x}}, r_{\mathrm{y}}, r_{\mathrm{z}}\right)$ uniquely represents an eye position. Rotation vectors were derived from the voltages induced in scleral search coils. ${ }^{12}$ We computed the stereographic projection of the normalized gaze vector onto a tangent screen. For the Hess screen plots, this gives

$$
\begin{gathered}
\boldsymbol{b}^{e}=\frac{-r_{z}-r_{x} r_{y}}{1+r_{x}^{2}} \\
v^{e}=\frac{-r_{y}+r_{x} r_{z}}{1+r_{x}^{2}}
\end{gathered}
$$

where $b^{e}$ is the horizontal and $v^{e}$ the vertical component. ${ }^{13}$ Cyclotorsion $t$, which is not displayed on the Hess screen, is defined by

$$
t^{e}=r_{x}
$$

The angular velocity vector $\boldsymbol{\omega}$, which describes the instantaneous eye movement corresponding to the rotation vector $\mathbf{r}^{\mathrm{e}}(t)$, is given by ${ }^{14}$ :

$$
\boldsymbol{\omega}=\frac{2\left(\dot{\boldsymbol{r}}^{e}+\boldsymbol{r}^{e} \times \dot{\boldsymbol{r}}^{e}\right)}{1+\left|\boldsymbol{r}^{e}\right|^{2}}
$$

To compare the orientation of $\omega$ to the gaze direction, we normalized $\omega$ by $\mathbf{n}^{\omega}=\left(n_{x}, n_{y}, n_{z}\right)=\frac{\omega}{|\omega|}$, and also determined its stereographic projection:

$$
\begin{aligned}
\boldsymbol{b}^{\omega} & =\frac{-2 n_{y}}{1+n_{x}} \\
\boldsymbol{v}^{\omega} & =\frac{2 n_{z}}{1+n_{x}}
\end{aligned}
$$


For convenience, Hess screen charts were labeled in degrees:

$$
\begin{aligned}
& \boldsymbol{b}_{\mathrm{deg}}=\tan ^{-1}(\boldsymbol{b}) \frac{2 \cdot 180}{\pi} \\
& v_{\mathrm{deg}}=\tan ^{-1}(v) \frac{2 \cdot 180}{\pi}
\end{aligned}
$$

\section{Appendix B}

\section{Videos}

The following videos are available online at http://www. iovs.org/cgi/content/full/45/2/455/DC1.

Bielschowsky.mov. Documentation of dynamic Bielschowsky head-tilt test. First scene: three-dimensional turntable oscillating $\pm 35^{\circ}$ at $0.3 \mathrm{~Hz}$. Second scene: binocular and monocular video recording during oscillation of a patient with acquired left trochlear nerve palsy in right gaze. The paretic left eye was covered with a cyan filter blocking the red laser light but allowing video recording. Increasing hyperdeviation of the covered eye with intorsion. Third scene: computer animation of desaccaded original search-coil data of the same patient at the same gaze direction as in scene two. Red lines indicate instantaneous ocular rotation axes. Axis of the covered eye is shifted nasally relative to the axis of the fellow eye and away from the line of sight.
Example_Patient.mov (complementary to Fig. 4, but a different patient). Acquired right trochlear nerve palsy. Patient looking at nine different target directions during sinusoidal head roll with the palsied eye covered. Rotation axis of viewing eye roughly aligns with the line of sight. Rotation axis of covered eye deviates nasally relative to the axis of the fellow eye, but remains fairly stable.

Hess_Screen.mov (complementary to Figs. 1 and 4, but different patient). Reconstruction of Hess screen grid during dynamic Bielschowsky head-tilt testing of a congenital right trochlear nerve palsy. For every gaze direction, mean eye position during oscillation cycles was calculated for each chair position in steps of $1^{\circ}$. Covered right eye: red. Viewing left eye: blue.

Geometric_Model.mov (complementary to Fig. 7D). Computer animation of geometric two-step model simulating right trochlear nerve palsy. Left healthy eye (viewing): sinusoidal $\pm 10^{\circ}$ torsion about the line of sight in nine different gaze directions. Right affected eye (covered): rotation about a stable rotation axis (red line) deviated $15^{\circ}$ nasally relative to the axis of the healthy eye and not aligned with the line of sight.

Eyelab_Model.mov (complementary to Fig. 10). Computer animation of EyeLab model simulating right trochlear nerve palsy. Left viewing eye (healthy: model input): sinusoidal $\pm 10^{\circ}$ torsion about the line of sight at nine different gaze directions. Right covered eye (affected: model output): oscillating deviation of rotation axis (red line) toward the nose and vertical deviation of gaze as a function of torsion. 